\title{
Notes on some Lepidoptera Tortricidae from Central Asia
}

\section{P. Trematerra}

\section{Università degli Studi del Molise, Dipartimento di Scienze Animali, Vegetali e dell'Ambiente, Campobasso, Italy}

\begin{abstract}
Faunistic data of some Lepidoptera Tortricidae collected in mountainous localities of Kazakhstan, Kyrgyzstan and Turkmenija, are reported. The total number of species recorded is 69 ; some of them are of special biogeographical interest.
\end{abstract}

\section{Introduction}

In the present paper, Lepidoptera Tortricidae are reported to have been found among material collected in the mountainous localities of Kazakistan, Kirgizstan and Turkmenija. The specimens were taken in the period from 1974 to 2000 by colleagues Sergei V. Churkin (Moscow, Russia), Vidmandas Karalius, Povilas Ivinskis, Jan Miatleuski, and Aidas Saldaitis (Vilnius, Lithuania). Specimens were collected applying electrical lamps of different types. Part of the material was trapped during the daytime, and a few species were raised from larvae.

Microlepidoptera fauna of Central Asia is less known. Some parts of the region were not completely inventoried and studied. The present paper provides new data on the distribution of some Tortricidae (total number of species 69). Among other tortricids of particular interest were: Phtheochroa kenneli (Obraztsov), Cochylimorpha halophilana (Christoph), C. alternana (Stephens), C. emiliana (Kennel), C. pirizanica (Razowski), and C. woliniana (Schleich), Phalonidia contractana (Zeller), Agapeta zoegana (Linnaeus), Eugnosta lathoniana (Hübner), Cochylidia rupicola (Curtis) and C. moguntiana (Rössler), Eana samarcandae (Razowski), Cnephasia cupressivorana (Staudinger) and C. longana (Haworth), Clepsis moeschleriana

Correspondence: Pasquale Trematerra, Università degli Studi del Molise, Dipartimento di Scienze Animali, Vegetali e dell'Ambiente, via de Sanctis, 86100 Campobasso, Italy. E-mail: trema@unimol.it

Key words: Lepidoptera Tortricidae, faunistc records, Central Asia.

Received for publication: 23 February 2012.

Revision received: 14 March 2012.

Accepted for publication: 23 March 2012.

(C) Copyright P. trematerra, 2012

Licensee PAGEPress, Italy

Journal of Entomological and Acarological Research 2012; 44:e1

doi:10.4081/jear.2012.e1

This article is distributed under the terms of the Creative Commons Attribution Noncommercial License (by-nc 3.0) which permits any noncommercial use, distribution, and reproduction in any medium, provided the original author(s) and source are credited.
(Wocke) and C. praeclarana (Kennel), Endothenia hebesana (Walker), Celypha ermolenkoi Kostyuk, Pelochrista griseolana (Zeller) and $P$. medullana (Staudinger), Pammene fasciana (Linnaeus) (Razowski, 1979, 2002, 2003 and 2009; Kuznetsov, 1989; Brown, 2005; Alipanah, 2009; Trematerra, 2010a and 2010b).

\section{List of taxa Tortricidae}

\section{Subfamily Tortricinae}

Tribe Tortricini

Acleris scabrana ([Denis \& Schiffermüller], 1775)

MATERIAL EXAMINED. 1 female, European p. of Kazakistan, Dzhanibek env. Kandagash loc., 21-27.06.1999, Leg. Miatleuski J. and Karalius V.

DISTRIBUTION. W Europe to Russian Far East, Asia Minor and North America.

Tribe Cochylini

Phtheochroa inopiana (Haworth, [1811])

MATERIAL EXAMINED. 3 males, Kirgizstan, Suusamyr mts., Kokemeren r, 5 km N Kyzyl-0i, 1800 m, 24-25.06.2000, S. Churkin leg.; 1 male and 1 female, Kirgizstan, E Suusamyr mts., Kovjuksu r., 1650 m, Kyzyl-oi, 26.07.00, S. Churkin leg.

DISTRIBUTION. Entire Palaearctic, from Iberian Peninsula and British Islands to Japan.

Phtheochroa kenneli (Obraztsov, 1944)

MATERIAL EXAMINED. 1 male, Kazakistan S.W. Mangishlak pen. W. $45 \mathrm{Km}$ S.E. from Aktan, Karagije loc., 10.30.04.99, Miatleuski leg. DISTRIBUTION. From S Europe, S Ukraine, Crimea, lover Volga area, SE Urals and Caucasus (Dasghestan), Asia.

Cochylimorpha halophilana (Christoph, 1872)

MATERIAL EXAMINED. 1 male, Turkmenija Bachardeno r. kov Ata, 1981.IX.17, P. Ivinskis.

DISTRIBUTION. From Central SE Europe (lower Volga, S Ural mts.), Caucasus to Iran and Afghanistan.

Cochylimorpha asiana (Kennel, 1899)

MATERIAL EXAMINED. 1 male, Kirgizstan, S. Issyk-kul L., KadzhiSai v., 1.07.00, $1800 \mathrm{~m}$, S. Churkin leg.

DISTRIBUTION. SE Europe (Ukraine and Russia), Libya, Caucasus, Turkmenia and from Kazakhstan to C Asia and Mongolia, Iran, Afghanistan, China.

Cochylimorpha cultana (Lederer, 1855)

MATERIAL EXAMINED. 1 male, Turkménija, Kara-Kala r. Ai-Dere, 1985.V.8, P. Ivinskis.

DISTRIBUTION. In Europe known from Spain, S France and S Ural mts; widely distributed in Asia (W Turkestan to Alai, Shansi, Hoengshan in China) and N Africa (Algeria, Tunisia). 
Cochylimorpha alternana (Stephens, 1834)

MATERIAL EXAMINED. 1 male and 1 female, European p. of Kazakistan, Ryn-kum sandy steppe NW. Kandagash loc., 0517.06.1999, Miatleuski J. leg. and Karalius V. leg.

DISTRIBUTION. W Palaearctic, from France and British Islands eastward to Ural mts. and Asia Minor, also Libya.

Cochylimorpha emiliana (Kennel, 1919)

MATERIAL EXAMINED. 1 female, Turkménija, Ai-Dere, 1984.V.15, P. Ivinskis.

DISTRIBUTION. Known from East Tannuola, Mongolia.

Cochylimorpha pirizanica (Razowski, 1963)

MATERIAL EXAMINED. 1 female, Kirgizstan, S Issyk-Kul L., Ottuk v, 1650 m, 29.06.2000, S. Churkin leg.; 2 females, Kirgizstan (Fergana valley), $27 \mathrm{~km} \mathrm{S.0sh,} \mathrm{19.07.2000,} 1200 \mathrm{~m}$, S. Churkin leg.

DISTRIBUTION. Iran: Fars Province, Pirezan and Muk road.

Cochylimorpha woliniana (Schleich, 1868)

MATERIAL EXAMINED. 1 female, Kirgizstan, S. Issyk-kul L., KadzhiSai v., 1.07.00, $1800 \mathrm{~m}$, S. Churkin leg.

DISTRIBUTION. Known from Europe, mainly from its southern and median parts; C. woliniana luteola (Kuznetsov, 1975) in W Siberia and Mongolia.

Phalonidia latifasciana (Razowsky, 1970)

MATERIAL EXAMINED. 1 female, Turkménija, Ai-Dere, 1984.V.14, P. Ivinskis.

DISTRIBUTION. Europe: S Urals; C Asia: Kazakhstan.

Phalonidia contractana (Zeller, 1847)

MATERIAL EXAMINED. 1 female, Turkmenija Bachardeno r. kov Ata, 1981.IX.17, P. Ivinskis; 1 male, Kazakistan, S.E. Balcanas vill. Ili riv. env., 15-20.08.94, Saldaitis leg.; 1 male, Kirgizstan, S. Toktogul L., 15 km NE Karakul v., 1300-1400 m, 17.06.2000, S. Churkin leg.; 2 males, Kirgizstan, South Chatkal, 5 km. E Aflatun v., Karasu r., 1350 m, 19.06.2000, S. Churkin leg.

DISTRIBUTION. Widely distributed from Iberian Peninsula to Ural mts. and Greece. Also found in Asia Minor, Israel, Afghanistan and Kashmir, China (Xinjiang).

Phalonidia affinitana (Douglas, 1846)

MATERIAL EXAMINED. 1 female, European p. of Kazakistan, Rynkum sandy steppe NW. Kandagash loc., 05-17.06.1999, Miatleuski J. leg. and Karalius V. leg.

DISTRIBUTION. Known from W Europe to Ural mts. and Caucasus.

Agapeta hamana (Linnaeus, 1758)

MATERIAL EXAMINED. 1 male and 2 females, European p. of Kazakistan, Ryn-kum sandy steppe NW Kandagash loc., 0517.06.1999, Miatleuski J. and Karalius V. leg.; 2 females, European p. of Kazakistan, Dzhanibek env. Kandagash loc., 21-27.06.1999, Miatleuski J. and Karalius V. leg.; 1 female, Kirgizstan, S Issyk-Kul L., Ottuk v, 1650 m, 29.06.2000, S. Churkin leg.; 1 male and 1 female, Kirgizstan, E Suusamyr mts., Kovjuksu r., 1650 m, Kyzyl-oi, 26.07.00, S. Churkin leg.

DISTRIBUTION. W Palaearctic, excluding the southern territories, north to $66^{\circ}$ (Kazakhstan, Transcaucasia, C Asia, W \& S Siberia, Asia Minor, Iran, Afghanistan, Mongolia, W China and N India).
Agapeta zoegana (Linnaeus, 1767)

MATERIAL EXAMINED. 6 males, European p. of Kazakistan, Ryn-kum sandy steppe NW Kandagash loc., 05-17.06.1999, Miatleuski J. leg. and Karalius V. leg.

DISTRIBUTION. W Palaearctic species known from W Europe to Ural mts. and Asia Minor.

Eugnosta lathoniana (Hübner, 1800)

MATERIAL EXAMINED. 1 male, Kazakistan, S.W. Mangishlak pen. W. $45 \mathrm{Km}$ S.E. from Aktan, Karagije loc., 10.30.04.99, Miatleuski leg. DISTRIBUTION. S Europe from Iberian Peninsula to Peloponnese, from Germany to Ural mts. and Caucasus, Also Algeria, Asia Minor, Armenia.

Eupoecilia sanguisorbana (Herrich-Schäffer, 1860)

MATERIAL EXAMINED. 1 male, Kirgizstan, Suusamyr mts., Kokemeren r, 5 km N Kyzyl-0i, 1800 m, 24-25.06.2000, S. Churkin leg.

DISTRIBUTION. Europe excluding southern areas, Ural mts., W Kazakhstan, China (Heilongjiang).

Aethes xanthina (Falkovitsh, 1963)

MATERIAL EXAMINED. 1 female, Kirgizstan, Suusamyr mts., Kokemeren r, 5 km N Kyzyl-Oi, 1800 m, 24-25.06.2000, S. Churkin leg.; 1 female, Kirgizstan, E Suusamyr mts., Kovjuksu r., 1650 m., Kyzyl-oi, 26.07.00, S. Churkin leg; 1 female, Turkménija, Ai-Dere, 1984.V.13, P. Ivinskis.

DISTRIBUTION. SE Europe, (Ural mts.); also known from Turkmenia and Iran.

Aethes dilucidana (Stephens, 1852)

MATERIAL EXAMINED. 1 male and 1 female, Kirgizstan, South Chatkal, 5 km. E Aflatun v., Karasu r., 1350 m, 19.06.2000, S. Churkin leg.; 1 female, Kirgizstan, S Issyk-Kul L., Ottuk v, 1650 m, 29.06.2000, S. Churkin leg.

DISTRIBUTION. W Palaearctic species, from Europe to Algeria; S Siberia, Kazakhstan, Turkmenia (the literature data concerning Asia require re-examination).

Aethes bilbaensis (Rössler, 1877)

MATERIAL EXAMINED. 1 male, Kirgizstan, S Chatkal, 5 km E Aflatun v., Karasu r., 1350 m, 19.06.2000, S. Churkin leg.; 1 female, Kirgizstan, SE Toktogul L, Sargata v., 900-, 10.07.1999, 1100 m, S. Churkin leg.

DISTRIBUTION. W Palaearctic species, its area extends from NW Africa and Iberian Peninsula to Greece and S Ural mts., also known from Asia Minor, N Lebanon, Iran, Afghanistan and Turkmenia; Crimea, Caucasus, Transcaucasia and W Kazakhstan.

Aethes spirana (Kennel, 1899)

MATERIAL EXAMINED. 1 female, Turkménija, Kara-Kala r. Ai-Dere, 1985.V.8, P. Ivinskis.

DISTRIBUTION. Kazakhstan, Uzbekistan, Tadzhikistan, C Asia, Alexander mts., Iran.

Cochylidia rupicola (Curtis, 1834)

MATERIAL EXAMINED. 1 female, Turkménija, Ai-Dere, 1984.V.14, P. Ivinskis.

DISTRIBUTION. West Palaearctic species, recorded from Europe and Asia Minor.

Cochylidia moguntiana (Rössler, 1864)

MATERIAL EXAMINED. 3 males and 3 females, Kirgizstan, S. Issykkul L., Kadzhi-Sai v., 1800 m, 1.07.00, S. Churkin leg.

DISTRIBUTION. Europe from Spain to Balkan Peninsula, C Europe to Ural mts.; Siberia, Buryatia, Chita, Afghanistan, China. N Pakistan.

Cochylis posterana (Zeller, 1847)

MATERIAL EXAMINED. 1 female, Kirgizstan, Toktogul area, Karasù L., 2100 m, 16.06.2000, S. Churkin leg.; 1 male, Kirgizstan, S Issyk- 
Kul L., Ottuk v, 1650 m, 29.06.2000, S. Churkin leg; 2 females, Turkménija, Ai-Dere, 1984.V.15, P. Ivinskis; 1 male and 3 females, Turkménija, Kara-Kala r. Ai-Dere, 1985.V.7-8, P. Ivinskis.

DISTRIBUTION. Europe, Ukraine, European Russia east as far as Transkaspia.

Cochylis piana (Kennel, 1919)

MATERIAL EXAMINED. 1 male, Kirgizstan, Suusamyr mts., Kokemeren r, 5 km N Kyzyl-0i, 1800 m, 24-25.06.2000, S. Churkin leg.

DISTRIBUTION. Kazakhstan, Tadzhikistan, Uzbekistan, Mongolia, Iran, Afghanistan, C Asia, Djarkent, China (Shaanxi, Sinjiang).

Cochylis amoenana (Kennel, 1899)

MATERIAL EXAMINED: 1 male and 2 females, Kirgizstan, Toktogul area, Karasù L., 2100 m, 16.06.2000, S. Churkin leg.

DISTRIBUTION. Asia Minor, $\mathrm{N}$ Caucasus, Transcaucasia, Tadzhikistan, Afghanistan, Pakistan, Iran.

\section{Tribe Cnephasiini}

Eana samarcandae (Razowski, 1958)

MATERIAL EXAMINED: 1 male and 1 female, Kirgizstan, S. Toktogul L., 15 km NE Karakul v., 1300-1400 m, 17.06.2000, S. Churkin leg.; 4 males and 8 females, Kirgizstan, South Chatkal, 5 km E Aflatun v., Karasu r., 1350 m, 19.06.2000, S. Churkin leg. DISTRIBUTION. Recorded from Samarkand (Uzbekistan).

Cnephasia asiatica (Kuznetsov, 1956)

MATERIAL EXAMINED: 2 males, Turkménija, Ai-Dere, 1974.V.11, P. Ivinskis; 2 females, Turkménija, Ai-Dere, 1984.V.8-15, P. Ivinskis; 1 female, Turkménija, Kara-Kala r. Ai-Dere, 1985.V.8, P. Ivinskis; Turkménija, Ai-Dere, 1984.V.8, P. Ivinskis. DISTRIBUTION. Turkmenistan.

Cnephasia communana (Herrich-Schäffer, 1851)

MATERIAL EXAMINED. 3 males and 4 females, Turkménija, Ai-Dere, 1984.V.7-13, P. Ivinskis.

DISTRIBUTION. W Palaearctic species, from Iberian Peninsula to Karelia and Caucasus; Transcaucasia, Trans-Ural, S. Siberia, Kazakhstan and mountains of Turkmenia, also Libya and Asia Minor.

Cnephasia cupressivorana (Staudinger, 1871)

MATERIAL EXAMINED. 1 female, Kirgizstan, S Issyk-Kul L., Ottuk v, 1650 m, 29.06.2000, S. Churkin leg.; 1 female, Kirgizstan, Inn. TianShan, Naryn r. vall, Kazarman vic., 1400 m., 21.07.2000, S. Churkin leg.

DISTRIBUTION. Southern Europe from Balkan Peninsula to Rumania, Austria and Asia Minor.

Cnephasia longana (Haworth, [1811])

MATERIAL EXAMINED. 1 female, Kirgizstan, SE Toktogul L, Sargata v., $900-10.07 .1999,1100 \mathrm{~m}$, leg. S. Churkin.

DISTRIBUTION. Known from Europe to Mediterranean and east to W European Russia.

Tribe Archipini

Epagoge grotiana (Fabricius, 1781)

MATERIAL EXAMINED. 3 males, European p. of Kazakistan Dzhanibek env. Kandagash loc., 21-27.06.1999, Miatleuski J. leg. and Karalius V. leg.

DISTRIBUTION. From W and N Europe to Ukraine and S Ural mts., Trans-Ural and Asia Minor.

Periclepsis cinctana (Denis \& Schiffermüller, 1775)

MATERIAL EXAMINED. 2 males, European p. of Kazakistan Dzhanibek env. Kandagash loc. 21-27.06.1999. Leg. Miatleuski J., Karalius V.

DISTRIBUTION. Known all over Europe; the data from Asia require confirmation.
Archips betulanus (Hübner, [1787])

MATERIAL EXAMINED. 1 female, Kirgizsky, Mts S slopes, Karakol r., Balyktyr., 2300 m, 13.07.1999, S. Churkin leg.

DISTRIBUTION. Palaearctic species, probably excluding the south.

Archips rosanus (Linnaeus, 1758)

MATERIAL EXAMINED. 2 males, Kirgizstan, Tash-kumyr v., 1000 m, 18.06.2000, S. Churkin leg.; 1 female, Kirgizstan, S Chatkal, 5 km E Aflatun v., Karasu r., 1350 m, 19.06.2000, S. Churkin leg.

DISTRIBUTION. Palaearctic species; introduced in North America.

Pandemis cinnamomeana (Treitschke, 1830)

MATERIAL EXAMINED. 2 females, Kirgizstan, Toktogul area, Karasù L., 2100 m, 16.06.2000, S. Churkin leg.

DISTRIBUTION. W Europe to Ural mts., Siberia, Mongolia, Russian Far East, NW China, Korea, Japan.

Pandemis chondrillana (Herrich-Schäffer, 1860)

MATERIAL EXAMINED. 8 males and 1 female, European p. of Kazakistan, Ryn-kum sandy steppe NW Kandagash loc., 0517.06.1999, Miatleuski J. leg., Karalius V. leg.; 1 male, European p. of Kazakistan, Dzhanibek env. Kandagash loc., 21-27.06.1999, Miatleuski J. leg., Karalius V. leg.; 1 female, Kirgizstan, S. Toktogul L., 15 km NE Karakul v., 1300-1400 m, 17.06.2000, S. Churkin leg. DISTRIBUTION. Europe, Ukraine and southern areas of European Russia; also recorded in Asia Minor, Iran, Afghanistan and in Kazakhstan through Siberia to Mongolia, C Asia and NW China.

Clepsis moeschleriana (Wocke, 1862)

MATERIAL EXAMINED: 1 male, Kirgizstan, S Issyk-Kul L., Ottuk v., 1650, 29.06.2000, S. Churkin leg.

DISTRIBUTION. A boreo-alpine species. Polar Ural, Altai mts.; in Nearctics spread from Labrador to Alaska, known from other parts of Canada and USA.

Clepsis praeclarana (Kennel, 1899)

MATERIAL EXAMINED: 1 female, Kirgizstan, S. Issyk-kul L., KadzhiSai v., 1.07.00, $1800 \mathrm{~m}$, S. Churkin leg.

DISTRIBUTION. SE Europe, Georgia, SW Siberia, Saisan and Mongolia.

Clepsis neglectana (Herrich-Schäffer, 1851)

MATERIAL EXAMINED. 5 males and 1 female, Kirgizstan, Suusamyr mts., Kokemeren r, 5 km N Kyzyl-0i, 1800 m, 24-25.06.2000, S. Churkin leg.; 1 male, Kirgizstan, S Issyk-Kul L., Ottuk v, 1650 m, 29.06.2000, S. Churkin leg.

DISTRIBUTION. Widely distributed in Europe; also known from $\mathrm{N}$ Africa, Cyprus and Lebanon; in C Asia reaching Kirgizstan and Tuva.

Clepsis trifasciata (Trematerra, 2010)

MATERIAL EXAMINED. 1 male, holotype, labelled as follows: Kirgizstan, S Issik-Kul L., Kadzhi-Sai v., 1.07.2000, 1800 m, S. Churkin leg.; paratype, 1 male with same label (Trematerra, 2010). DISTRIBUTION. Known from Kirgizstan.

\section{Subfamily Olethreutinae}

Tribe Bactrini

Bactra lacteana Caradja, 1916

MATERIAL EXAMINED. 1 male, European p. of Kazakistan, Dzhanibek env. Kandagash loc., 21-27.06.1999, Miatleuski J. leg. and Karalius V. leg.

DISTRIBUTION. Europe except for northern areas, Caucasus, Kazakhstan, W Mongolia, Russia (from S Siberia to Primorsk), China, Japan.

Bactra bactrana (Kennel, 1901)

MATERIAL EXAMINED. 1 male, Kirgizstan, Suusamyr mts., Kokemeren r, 5 km N Kyzyl-Oi, 1800 m, 24-25.06.2000, S. Churkin leg. 
DISTRIBUTION. Canary Islands, S Spain, Italy, Marocco, Algeria, Malta, Egypt, Asia Minor, Arabia, Mesopotamia, Iran, Iraq, Caucasus, Afghanistan, Caspian area, Tadzhikistan, Uzbekistan, Pakistan, India, and Ethiopia.

\section{Tribe Olethreutini}

\section{Endothenia hebesana (Walker, 1863)}

MATERIAL EXAMINED. 1 female, European p. of Kazakistan, Dzhanibek env. Kandagash loc., 21-27.06.1999, Miatleuski J. leg. and Karalius V. leg. DISTRIBUTION. From Scandinavia, Finland, European NW Russia, W Siberia and S Primorsk, NW China, Nearctic region.

Celypha rufana (Scopoli, 1763)

MATERIAL EXAMINED. 1 female, European p. of Kazakistan, Dzhanibek env. Kandagash loc., 21-27.06.1999, Miatleuski J. leg., Karalius V. leg.

DISTRIBUTION. N C and E Europe, Transcaucasia, S Siberia, Tuva, Cisbaikal, and Korea.

Celypha ermolenkoi Kostyuk, 1980

MATERIAL EXAMINED. 1 female, Kirgizstan, E Suusamyr mts., Kovjuksu r., 1650 m, Kyzyl-oi, 26.07.00, S. Churkin leg.

DISTRIBUTION. Ukraine (Crimea).

Celipha cespitana (Hübner, [1814-1817])

MATERIAL EXAMINED. 2 males, Kirgizstan, Toktogul area, Karasù L., $2100 \mathrm{~m}, 16.06 .2000$, S. Churkin leg.; 1 male and 3 females, Kirgizstan, South Chatkal, 5 km. E Aflatun v., Karasu r., 1350 m, 19.06.2000, S. Churkin leg.; 10 males, Kirgizstan, Suusamyr mts., Kokemeren r, 5 km N Kyzyl-0i, 1800 m, 24-25.06.2000, S. Churkin leg.; 1 male, Inn. Tian-Shan Naryn r. vall, Kazarman vic., 1400 m., 21.07.2000, S. Churkin leg.

DISTRIBUTION. W Europe, to Ural mts., Transcaucasia, Asia Minor, Near East, Iran, Siberia, C Asia, Cisbaikal, Tuva, Primorsk, Manciuria, Korea, Japan, North America.

\section{Tribe Eucosmini}

Thiodia torridana (Lederer, 1859)

MATERIAL EXAMINED. 2 females, Kirgizstan, Toktogul area, Karasù L., 2100 m, 16.06.2000, S. Churkin leg.

DISTRIBUTION. Europe excluded N and SW parts, Ural mts., Transcaucasia, Asia Minor, Kazakhstan, Turkmenia, S Siberia, AmurZeya-area, Priamure, S Primorsk, S Sakhalin, Kunashir Islands.

Thiodia lerneana (Treitschke, 1835)

MATERIAL EXAMINED. 1 female, Kazakistan, S.E. Balcanas vill. Ili riv. env., 15-20.08.94, leg. Saldaitis; 3 females, Kirgizstan, Suusamyr mts., Kokemeren r, 5 km N Kyzyl-0i, 1800 m, 24-25.06.2000, S. Churkin leg.

DISTRIBUTION. Spain, Italy, Austria to Hungary and Balkan Peninsula, S E Europe, Transural, Kazakhstan, Turkmenia.

Thiodia citrana (Hübner, [1796-99])

MATERIAL EXAMINED. 1 female, European p. of Kazakistan, Rynkum sandy steppe NW Kandagash loc., 05-17.06.1999, Miatleuski J. leg. and Karalius V. leg.

DISTRIBUTION. Europe from Spain to Karelia, Lebanon, Caucasus, Transcaucasia, Asia Minor, Iran, Kazakhstan, Turkmenia, S Siberia, S. Primorsk, China, Japan.

Epinotia thapsiana (Zeller, 1847)

MATERIAL EXAMINED. 1 male, Kirgizstan, Suusamyr mts., Kokemeren r, 5 km N Kyzyl-0i, 1800 m, 24-25.06.2000, S. Churkin leg.

DISTRIBUTION. Canary Islands, southern and central areas of Europe to E European Russia, Transcaucasia, Asia Minor, Iran, S Siberia, Kazakhstan, Tadzhikistan, Turkmenia, Korea.
Pelochrista griseolana (Zeller, 1847)

MATERIAL EXAMINED. 1 female, European p. of Kazakistan, Dzhanibek env. Kandagash loc., 21-27.06.1999, Miatleuski J. leg. and Karalius V. leg.

DISTRIBUTION. Spain and Sicily.

Pelochrista medullana (Staudinger, 1879)

MATERIAL EXAMINED. 1 male, European p. of Kazakistan, Ryn-kum sandy steppe NW Kandagash, loc. 05-17.06.1999, Miatleuski J. leg. and Karalius V. leg.; 2 males, Kirgizstan, Toktogul area, Karasù L., 2100 m, 16.06.2000, S. Churkin leg.; 1 male, Kirgizstan, Suusamyr mts., Kokemeren r, 5 km N Kyzyl-0i, 1800 m, 24-25.06.2000, S. Churkin leg.

DISTRIBUTION. Balkan Peninsula, Europe, Asia Minor, Iran.

Pelochrista modicana (Zeller, 1847)

MATERIAL EXAMINED. 1 male, Turkmenija, Morgunovka, 1974.V.3, P. Ivinskis.

DISTRIBUTION. S E Europe, Asia Minor, Transcaucasia, Turkmenia, Kazakhstan.

Pelochrista arabescana (Eversmann, 1844)

MATERIAL EXAMINED. 11 males, Kazakistan, SW Mangishlak pen.W. $45 \mathrm{Km}$ SE from Aktan, Karagije loc., 10.30.04.99, Miatleuski leg.

DISTRIBUTION. Central, Southern and Eastern Europe, Caucasus, Transcaucasia, Iran, S Siberia, Kazakhstan, Turkmenia, Mongolia, China.

Eucosma lugubrana (Treitschke, 1830)

MATERIAL EXAMINED. 1 female, Kirgizstan, E Suusamyr mts., Kovjuksu r., 1650 m, Kyzyl-oi, 26.07.00, S. Churkin leg.

DISTRIBUTION. Europe, Kazakhstan.

Eucosma conterminana (Guenée, 1845)

MATERIAL EXAMINED. 1 female, European p. of Kazakistan, Dzhanibek env. Kandagash loc., 21-27.06.1999, Miatleuski J. and Karalius V. leg.; 2 males and 12 females, Kirgizstan, S Issyk-Kul L., Ottuk v, 1650 m, 29.06.2000, S. Churkin leg.; 2 females, (Kirgizstan) Fergana valley, 27 km S.0sh, 1200 m., 19.07.2000, S. Churkin leg. DISTRIBUTION. Europe, Caucasus, Ural mts, Transcaucasia, Asia Minor, Iran, Kazakhstan, C Asia, S Siberia, Mongolia, Priamure, S Primorsk, China.

Eucosma catoptrana (Rebel, 1903)

MATERIAL EXAMINED. 1 male, European p. of Kazakistan, Ryn-kum sandy steppe NW Kandagash loc., 05-17.06.1999, Miatleuski J. leg. and Karalius V. leg.

DISTRIBUTION. Europe, Kazakhstan.

Eucosma metzneriana (Treitschke, 1830)

MATERIAL EXAMINED. 1 male, Kirgizstan, S Issyk-Kul L., Ottuk v, 1650 m., 29.06.2000, S. Churkin leg.; 1 male, Kirgizstan, Inn. TianShan Naryn r. vall, Kazarman vic., 1400 m, 21.07.2000, S. Churkin leg.

DISTRIBUTION. Europe, S Russia, Ukraine, Karelia, Caucasus, Transcaucasia, Asia Minor, Iran, Kazakhstan, C Asia, Mongolia, Siberia, Amur-Zeya area, Priamure, S Primorsk, Kuanhsien, Korea, Japan.

Eucosma luciana (Kennel, 1919)

MATERIAL EXAMINED. 1 male, European p. of Kazakistan, Ryn-kum sandy steppe NW Kandagash loc., 05-17.06.1999, Leg. Miatleuski J. and Karalius V.

DISTRIBUTION. E Europe, Transcaucasia, S Siberia, Mongolia Russian Far East, NW China, Japan.

Eucosma pupillana (Clerck, 1759)

MATERIAL EXAMINED. 10 males and 9 females, European p. of Kazakistan, Ryn-kum sandy steppe NW Kandagash loc., 0517.06.1999, Miatleuski J. leg. and Karalius V. leg.; 2 males and 1 female, European p. of Kazakistan, Dzhanibek env. Kandagash loc., 
21-27.06.1999, Miatleuski J. leg. and Karalius V. leg.; 1 male, Kirgizstan, S. Toktogul L., 15 km NE Karakul v., 1300-1400 m, 17.06.2000, S. Churkin leg.; 2 males, Kirgizstan, South Chatkal, 5 km. E Aflatun v., Karasu r., 1350 m, 19.06.2000, S. Churkin leg.; 1 female, Kirgizstan, S Issyk-Kul L., Ottuk v, 1650 m, 29.06.2000, S. Churkin leg.

DISTRIBUTION. Europe, Ural mts., Asia Minor, Iran, Kazakhstan, Tadzhikistan.

Eucosma ephedrana (Christoph, 1877)

MATERIAL EXAMINED. 1 male, Kirgizstan, S. Issyk-kul L., Kadzhi-Sai v., 1.07.00, $1800 \mathrm{~m}$, S. Churkin leg.

DISTRIBUTION. Transcaucasia, C Asia, Iran, Afghanistan.

Eucosma clarescens (Kuznetsov, 1964)

MATERIAL EXAMINED. 1 female, European p. of Kazakistan, Rynkum sandy steppe NW Kandagash loc., 05-17.06.1999, Miatleuski J. leg. and Karalius V. leg.

DISTRIBUTION. Europe, Kazakhstan, Mongolia.

Gypsonoma oppressana (Treitschke, 1835)

MATERIAL EXAMINED. 1 male, Kirgizstan, E Suusamyr mts., Kovjuksu r., 1650 m, Kyzyl-oi, 26.07.00, S. Churkin leg. DISTRIBUTION. Europe, Trascaucasia, Kazakhstan, Tadzhikistan.

Epiblema foenellum (Linnaeus, 1758)

MATERIAL EXAMINED. 1 female, Kirgizstan, South Chatkal, 5km. E Aflatun v., Karasu r., 1350 m, 19.06.2000, S. Churkin leg.

DISTRIBUTION. Known from Palaearctic and Oriental regions.

Epiblema junctanum (Herrich-Schäffer, 1856)

MATERIAL EXAMINED. 1 female, European p. of Kazakistan, Rynkum sandy steppe NW Kandagash loc., 05-17.06.1999, Miatleuski J. leg. and Karalius V. leg.

DISTRIBUTION. Europe, Asia Minor, Iran, C Asia, Kazakhstan, Siberia, Priamure, S Primorsk.

Notocelia cynosbatella (Linnaeus, 1758)

MATERIAL EXAMINED. 3 males, Turkménija, Kara-Kala r. Ai-Dere, 1985.V.8, P. Ivinskis; Turkménija, Ai-Dere, 1984.V.14-15, P. Ivinskis.

DISTRIBUTION. Europe, Ural mts., Caucasus, Asia Minor, Near East, Iran, Trascaucasia, Kazakhstan, Siberia, Amur-Zeya area Priamure, S Primorsk, S Sakhalin.
Tribe Grapholitini

Cydia pomonella (Linnaeus, 1758)

MATERIAL EXAMINED. 1 male and 1 female, European p. of Kazakistan, Dzhanibek env. Kandagash loc., 21-27.06.1999, Miatleuski J. leg. and Karalius V. leg.

DISTRIBUTION. W Palaearctic, worldwide (introduced).

Pammene fasciana (Linnaeus, 1761)

MATERIAL EXAMINED. 1 female, Azerbaidän, Lenkoran, Aurora, 1987.VIII.4, P. Ivinskis.

DISTRIBUTION. Europe, Karelia, Ukraine.

\section{References}

ALIPANAH H., 2009 - Synopsis of the Cochylini (Tortricidae: Tortricinae: Cochylini) of Iran, with the description of a new species. - Zootaxa, 2245: 1-31.

BROWN J.W., 2005 - Tortricidae (Lepidoptera). In: World Catalogue of Insects 5. - Apollo Book Aps, Stenstrup, Denmark: 1-741.

KUZNETSOV V.I., 1989 - Family Tortricidae (Olethreutidae, Cochylidae) - Tortricid Moths. In: Medvedev G.S., Keys to the insects of the European Part of the USSR, Volume IV, Lepidoptera, Part I. - E.J. Brill, Leiden, The Netherlands: 279-991.

RAZOWSKI J., 1979 - Revision of the Genus Clepsis Guenée (Lepidoptera, Tortricidae). Part I. - Acta Zool. Cracov. XXIII (8): 101198.

RAZOWSKI J., 2002 - Tortricidae (Lepidoptera) of Europe, Volume 1, Tortricinae and Chlidanotinae. - Frantisek Slamka, Bratislava: 1247.

RAZOWSKI J., 2003 - Tortricidae of Europe. Volume 2. Olethreutinae. Frantisek Slamka, Bratislava: 1-301.

RAZOWSKI J., 2009 - Tortricidae of the Palaearctic Region, Volume 2, Cochylini. - Frantisek Slamka, Bratislava: 1-195.

TREMATERRA P., 2010a - Clepsis trifasciata sp. n. with notes on some Lepidoptera Tortricidae from Kirgizstan. - J. Entomol. Acarol. Res. Ser. II 42 (1): 1-10.

TREMATERRA P., 2010b - Lepidoptera Tortricidae from SE European Russia with description of Ceratoxanthis saratovica sp. n. - J. Entomol. Acarol. Res. Ser. II 42 (1): 19-26. 